\section{Assessment of patient- reported outcome and quality of life improvement following surgery for epiphora}

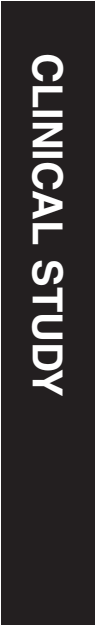

${ }^{1}$ Department of Ophthalmology, Royal Berkshire Hospital, Reading, UK

${ }^{2}$ Department of Ophthalmology, King Edward VII Hospital, Windsor, UK

Correspondence: Z Sipkova, Department of Ophthalmology, Royal Berkshire NHS Foundation Trust, Craven Road, Reading RG1 5AN, UK Tel: +44 (0)118 322 5111; Fax: $+44(0) 1183228148$. E-mail: zuzana.sipkova@ doctors.org.uk

Received: 30 November 2016

Accepted in revised form: 12 May 2017 Published online: 16 June 2017 +100 (maximal benefit).
Z Sipkova ${ }^{1,2}$, O Vonica $^{1,2}$, O Olurin ${ }^{2}$, EE Obi ${ }^{1,2}$ and AR Pearson ${ }^{1,2}$

\begin{abstract}
Purpose To assess and compare the subjective improvement in symptoms and quality of life in adult patients who underwent commonly performed oculoplastic

Conclusion Patients derived significant improvement in symptoms and health-related quality of life benefit following all surgical interventions for epiphora.
\end{abstract} surgical interventions to treat epiphora. Materials and methods A prospective study was undertaken involving all adult patients undergoing dacryocystorhinostomy (DCR), lid tightening (lateral tarsal strip or lateral wedge resection), and punctoplasty surgery at our institution. We assessed severity of epiphora preoperatively using the Munk score. At 3 months postoperatively, all patients were sent postal questionnaires comprising of Munk score, 'social impact score' from validated Lac- $Q$ questionnaire ranging from 0 (no impact) to 5 (maximal negative impact) and Glasgow Benefit Inventory (GBI) score, ranging from -100 (maximal detriment) to

Results A total of 134 questionnaires were sent with an overall response rate of $74.6 \%$. For the purpose of data analysis, patients were divided into four groups: DCR, lid tightening, punctoplasty, and combined group (lid tightening plus punctoplasty). There was statistically significant improvement in subjective epiphora postoperatively, as assessed by Munk score $(P<0.001)$ in all groups. The total GBI scores were +42.67 (95\% CI: 33.42-51.91) for DCR, +19.65 (95\% CI: 10.33-28.97) for lid tightening, +16.06 (95\% CI: 2.65-29.48) for punctoplasty, and +26.53 (95\% CI: 13.15-39.90) for the combined group, demonstrating a positive change in health status for all groups. There was negative correlation between total GBI and post-operative Munk scores $(r=-0.58$, $P<0.001$ ), and positive correlation between Lac-Q and Munk scores $(r=0.65, P<0.001)$.
Eye (2017) 31, 1664-1671; doi:10.1038/eye.2017.120; published online 16 June 2017

\section{Introduction}

The patient's perspective in assessment of success of a healthcare intervention has become increasingly important in recent years. With growing budget pressures within the healthcare systems, demonstrating quality of life (QoL) benefit is useful to justify the provision of services. Within oculoplastic practice, clinical assessment of interventions for epiphora can be subjective and anatomical success does not necessarily ensure functional improvement. Patient-reported outcome measures (PROMs) therefore offer a means of demonstrating benefit of these procedures.

Several studies have assessed patientperceived benefit and QoL improvement following dacryocystorhinostomy (DCR) surgery for nasolacrimal duct obstruction (NLDO), mostly using the PROM questionnaire called Glasgow Benefit Inventory (GBI). ${ }^{1-8}$

Ramey et $a l^{9}$ have recently performed a systematic review of all oculoplastic PROMs and have been able to identify only two validated lacrimal surgery questionnaires, the Nasolacrimal Duct Obstruction Symptom Score $(\mathrm{NLDO}-\mathrm{SS})^{10}$ and Lac-Q questionnaire, ${ }^{11}$ both specific to DCR surgery for NLDO. Currently, there is no information on the value of interventions for epiphora other than DCR; and there is no specific PROM that has been validated for assessment of outcomes following surgical interventions for epiphora other than DCR. 
In this prospective study, we aimed to assess and compare the subjective success and QoL improvement following three commonly performed oculoplastic operations for epiphora: DCR, lower lid tightening, and punctoplasty using PROMs.

\section{Materials and methods}

This was a prospective study involving adult patients undergoing surgical interventions for epiphora. The patients attended specialist lacrimal clinics under the care of a single consultant oculoplastic surgeon (AP) between 1st October 2014 and 31st October 2015. Patients undergoing endonasal or external DCR for primary acquired NLDO, lateral tarsal strip or lateral wedge resection for lid laxity or ectropion, and punctoplasty (posterior ampullotomy) for punctal stenosis were included. Exclusion criteria included previous lacrimal surgery, canalicular obstruction, and age younger than 18 years.

Preoperatively, on the day of surgery, all patients were asked to rate subjectively their epiphora severity based on the $0-4$ scale developed by Munk et al ${ }^{12}$ (Figure 1a), ranging from 0 (no watering) to 4 (constant watering).

At 3 months post surgery, each patient was sent a postal questionnaire (Supplementary content) comprising three sets of questions: the Munk score, the five questions related to 'social and lifestyle impact of tear duct problem' from the Lac-Q questionnaire (Figure 1b), and the GBI questionnaire (Figure 1c), along with an information leaflet and stamped addressed envelope.

The GBI, originally developed by Robinson et $a l^{13}$ for assessment of patient outcomes following otorhinolaryngological procedures, has been validated for use following oculoplastic procedures, including DCR, $, 2,6,7,10$ ptosis, ${ }^{14,15}$ ectropion and entropion surgery, ${ }^{5}$ and botulinum toxin for blepharospasm. ${ }^{16}$ As described in detail elsewhere, ${ }^{5}$ it is a post-interventional questionnaire, consisting of 18 questions with responses scored on a five-point Likert scale. In addition to questions measuring general perception of well-being (12 questions), change in social and physical health is also assessed with three questions each, and both the total and subscale scores are reported on a scale of - 100 (maximum detriment) through zero (no benefit/change) to +100 (maximum benefit).

One of the main limitations of the GBI is that it is a general QoL questionnaire and does not assess ocular or lacrimal symptoms specifically. We therefore included the Munk symptom score and Lac-Q questionnaire as part of the post-operative assessment. The Lac-Q (Figure 1b) has two sections, the first consisting of five questions to assess social impact of disease (range $0=$ no negative impact to $5=$ maximum negative impact), the second assessing four different potential lacrimal symptoms (watering, pain, stickiness, and lump) associated with NLDO for each eye. ${ }^{11}$ As only the score for watering was relevant to our study, and was included in the Munk score, the symptom score of the Lac-Q questionnaire was excluded and only the social impact score was used.

Verbal consent was obtained before proceeding with the questionnaire preoperatively, and the study was conducted in accordance with the ethical standards of the Declaration of Helsinki.

The GBI questionnaires were analysed as recommended by the GBI manual, ${ }^{17}$ and data analysis was performed using Microsoft Excel (Microsoft Corporation, Redmond, WA, USA). Correlations between variables were assessed with Spearman's correlation coefficient. For patients who had bilateral surgery, either right or left eye Munk score values were randomly selected using Excel randomisation function. Differences were regarded as statistically significant if $P$-value was $<0.05$.

\section{Results}

A total of 134 questionnaires were sent with 100 questionnaires returned, representing an overall response rate of $74.6 \%$. Five questionnaires were excluded because of incomplete details. Overall, the mean age of patients at the time of surgery was 69.5 years (range: 18-93). For the purpose of data analysis, patients were divided into four groups: DCR, lid tightening, punctoplasty, and combined group (lid tightening plus punctoplasty) with results summarised in Table 1. In the DCR group, there were 23 patients who had endonasal and two patients who had external DCR. All DCR patients had silicone stents inserted that were routinely removed at 4 weeks.

There was a statistically significant benefit perceived in all four groups postoperatively in terms of reduction in self-reported severity of epiphora as assessed by Munk score.

The total GBI scores of patients in the DCR, lid tightening, punctoplasty, and combined groups were +42.67 (95\% CI: 33.42-51.91), +19.65 (95\% CI: 10.3328.97), +16.06 (95\% CI: $2.65-29.48)$, and +26.53 (95\% CI 13.15-39.90), respectively, demonstrating a positive change in health status for all intervention groups. The distribution of total GBI scores for each patient group is demonstrated in Figure 2. Figure 3 shows GBI subscale scores, including total, general, physical and social, for the different interventions.

Comparing the GBI reported QoL change and the severity of epiphora for all patients, the reported improvement in QoL was significantly correlated with less severe levels of watering postoperatively $(r=-0.58$, $P<0.001$; Figure 4a). Similarly, the reported improvement 


\begin{tabular}{|l|c|}
\hline Epiphora severity & Munk score \\
\hline No watering & 0 \\
\hline Occasional watering requiring dabbing less than twice a day & 1 \\
\hline Watering requiring dabbing 2-4 times a day & 2 \\
\hline Watering requiring dabbing 5-10 times a day & 3 \\
\hline Watering requiring dabbing more than 10 times a day or constant watering & 4 \\
\hline
\end{tabular}

b

Social impact score (one point for each, $\max$ score $=5$ )

Which of these statements is true about the tear duct problem overall in the last 8 weeks?

1. Friends/family have commented about the watery eye problen

2. The watery eye problem has caused embarrassment in company

3. The watery/sticky eye problem has interfered with everyday activity like reading and driving

4. The vision is sometimes blurred because of the watery/sticky eye problem

5. Medical attendance: visit to family doctor or eye hospital because of the tear duct problem

Lacrimal symptom score ( $\max$ score $=14$ for each eye)

For each of the 4 problems, mark next to the statement which best describes the situation over the last 8 weeks

A. Watery eye

1. No watery eye problem

2. The eye waters occasionally, mainly outdoors

3. Troublesome watering of the eyes indoors and outdoors, some days

4. Troublesome watering of eyes most days

5. Troublesome watering of the eye everyday

B. Pain in and around the eye, soreness of eyelids

1. No pain

2. Some pain or soreness, but has not sought medical advice or treatmen

3. Pain or soreness, has used prescription eye drops

4. Painful or swollen (lacrimal abscess) requiring antibiotics or surgical drainage

C. Sticky eye

1. No problem with sticky eye

2. The eye is sometimes sticky in the mornings

3. The eye is sticky everyday in the mornings

4. The eye has sticky or mucous discharge throughout the day

5. There is infected discharge leaking through the skin of the lower eyelid (fistula)

D. Swelling or lump at the medial canthus (mucocele)

1. No swelling or lump

2. Swelling present, but only intermittently

3. Swelling present all the time

\begin{tabular}{ccc} 
last 8 weeks & & Right eye \\
$\square$ & 0 & $\square$ \\
$\square$ & 1 & $\square$ \\
$\square$ & 2 & $\square$ \\
$\square$ & 3 & $\square$ \\
$\square$ & 4 & $\square$ \\
Left eye & & Right eye \\
$\square$ & 0 & $\square$ \\
$\square$ & 1 & $\square$ \\
$\square$ & 2 & $\square$ \\
$\square$ & 4 & $\square$ \\
Left eye & & Right eye \\
$\square$ & 0 & $\square$ \\
$\square$ & 1 & $\square$ \\
$\square$ & 2 & $\square$ \\
$\square$ & 3 & $\square$ \\
$\square$ & 4 & $\square$ \\
Left eye & & Right eye \\
$\square$ & 0 & $\square$ \\
$\square$ & 1 & $\square$ \\
$\square$ & 2 & $\square$ \\
\hline & & $\square$ \\
\hline
\end{tabular}

C

For each question patients are asked to score the answer on a 5 point Likert scale:

$\begin{array}{ll}1 & \text { Much worse } \\ 2 & \text { A little or somewhat worse } \\ 3 & \text { No change } \\ 4 & \text { A little or somewhat better } \\ 5 & \text { Much better }\end{array}$

\section{Question}

Score

General

Subscale

Have the results of your operation affected the things you can do?

2. Have the results of your operation made your overall life better or worse?

3. Since your operation have you felt more or less optimistic about the future?

4. Since your operation do you have more or less self-confidence?

5. Since your operation do you feel better or worse about yourself?

6. Since your operation have you found it easier or harder to deal with company?

7. Since your operation do you feel more or less confident about job opportunities?

8. Since your operation do you feel more or less embarrassed when with a group of people?

9. Since your operation do you feel more or less self-conscious?

10. Since your operation are you more or less inconvenienced by your (specific) problem?

11. Since your operation have you been able to participate in more or fewer social situations?

12. Since your operation have you been more or less inclined to withdraw from social situations?

13. Since your operation do you feel you have more or less support from your friends?

14. Since your operation do you feel you have more or less support from your family?

15. Since your operation are there more or fewer people who really care about you?

16. Since your operation have you been to your doctor, or any reason, more or less often?

17. Since your operation have you had to take more or less medicine, for any reason?

18. Since your operation have you been more or less inconvenienced by your other health problems?

.

Social

Physical

Subscale

Figure 1 (a) Munk score. ${ }^{12}$ (b) Lac-Q questionnaire. ${ }^{11}$ (c) GBI questionnaire. ${ }^{5}$ 
Table 1 Summary of results for all intervention groups

\begin{tabular}{|c|c|c|c|c|}
\hline Summary of results & $D C R$ & Lid tightening & Punctoplasty & Combined \\
\hline Response rate & $73.2 \%$ & $75 \%$ & $71.9 \%$ & $80 \%$ \\
\hline Mean age & 63.2 & 74 & 53.8 & 79.1 \\
\hline Number of patients & 25 & 27 & 23 & 20 \\
\hline Male & 6 & 17 & 2 & 13 \\
\hline Female & 19 & 10 & 21 & 7 \\
\hline Number of procedures & 27 & 46 & 39 & 34 \\
\hline \multicolumn{5}{|l|}{ Munk score } \\
\hline Mean pre-op & 3.56 & 3.43 & 3.51 & 3.50 \\
\hline $95 \%$ CI pre-op & $3.24-3.87$ & $3.13-3.74$ & $3.25-3.78$ & $3.24-3.76$ \\
\hline Mean post-op & 0.48 & 1.89 & 1.54 & 1.15 \\
\hline \multirow[t]{2}{*}{$95 \%$ CI post-op } & $0.20-0.76$ & $1.50-2.29$ & $1.05-2.03$ & $0.66-1.63$ \\
\hline & $P<0.001$ & $P<0.001$ & $P<0.001$ & $P<0.001$ \\
\hline Lac-Q score post op (mean) & 1.08 & 1.70 & 1.64 & 1.21 \\
\hline \multicolumn{5}{|l|}{ GBI total score } \\
\hline Mean & 42.67 & 19.65 & 16.06 & 26.53 \\
\hline Median & 33.33 & 22.22 & 22.20 & 23.61 \\
\hline Standard deviation & 4.48 & 4.53 & 6.47 & 6.39 \\
\hline Minimum & 11.10 & -50 & -55.56 & -19.44 \\
\hline Maximum & 97.22 & 75 & 72.22 & 94.44 \\
\hline Lower 95\% CI & 33.42 & 10.33 & 2.65 & 13.15 \\
\hline Upper 95\% CI & 51.91 & 28.97 & 29.48 & 39.90 \\
\hline Time post-op (mean, days) & 119.08 & 130.04 & 116 & 100.15 \\
\hline
\end{tabular}

Note: 'combined' refers to lid tightening+puctoplasty.

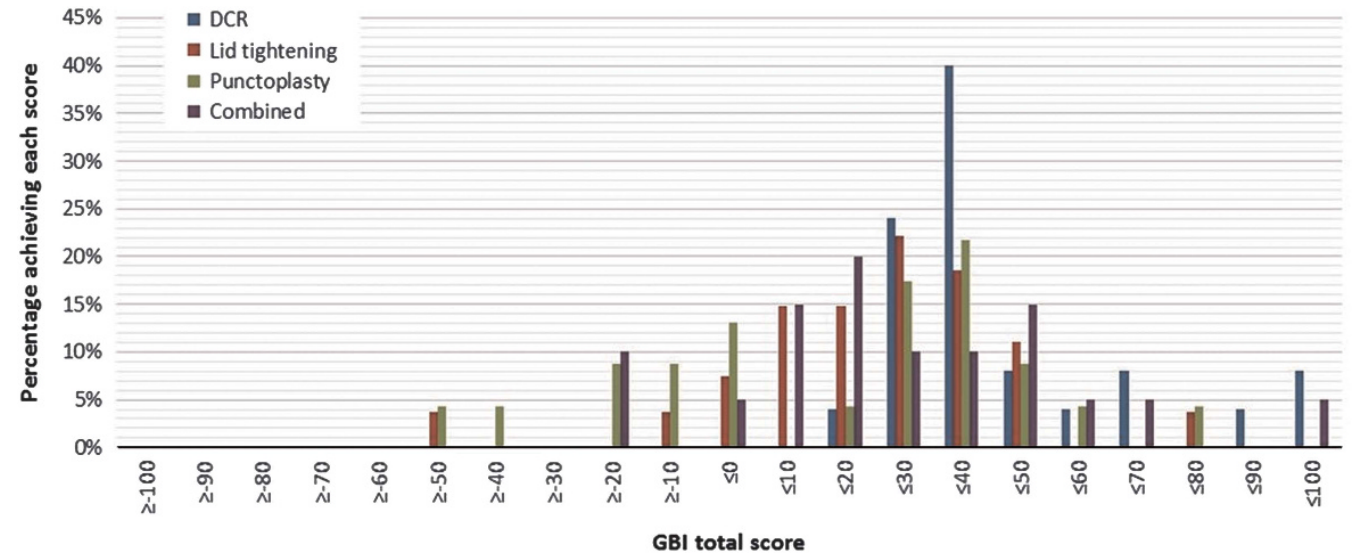

Figure 2 Graph comparing total GBI score distribution for different surgical intervention groups.

in QoL was significantly correlated with the amount of improvement in watering following the procedure $(r=0.57, P<0.001$; Figure $4 b)$.

Comparing the Lac-Q post-operative social impact score with the severity of epiphora for all patients, lower levels of post-operative watering and the amount of improvement in watering were both significantly correlated with lower social impact scores $(r=0.65$,
$P<0.001$; Figure $4 \mathrm{c})$ and $(r=0.50, P<0.001$; Figure $4 \mathrm{~d})$, respectively.

The GBI QoL and the Lac-Q social impact scores show significant correlation between the subjective improvement in QoL and lower social impact reported by patients $(r=-0.50, P<0.001$; Figure $4 \mathrm{e})$, indicating good consistency between the results of these two questionnaire scores. 


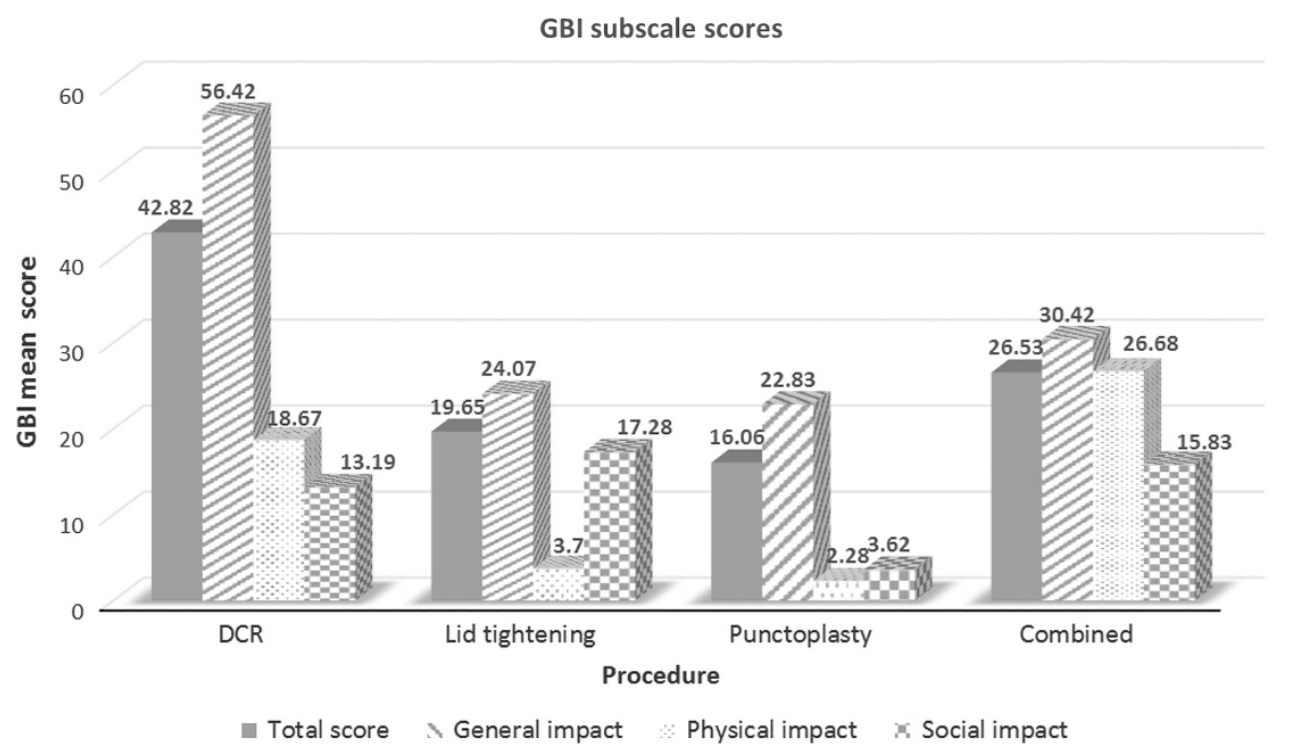

Figure 3 GBI subscale scores for each group.

\section{Discussion}

Epiphora, defined as overflow of tears from the eye, can have a significant impact on QoL, causing discomfort, embarrassment, and blurring of vision. Kafil-Hussain et $a l^{18}$ found that patients with epiphora suffer greater visual handicap in multiple areas of activities of daily living compared with the patients awaiting a second cataract surgery. Therefore, improving patient's QoL and symptoms are key objectives for surgery to treat epiphora.

Currently, questionnaire-based patient-reported outcome measures for procedures for epiphora are limited to DCR surgery. Several studies have assessed patient benefit following DCR surgery using the generic GBI questionnaire, which has been validated for DCR surgery and shown to be sensitive to change following an intervention. ${ }^{13} \mathrm{GBI}$ is not procedure-specific and therefore also permits comparison across different interventions, and can differentiate between successful and unsuccessful procedures. $^{7}$

However, Mistry et al ${ }^{11}$ argued that generic QoL questionnaires, such as GBI, may be of limited use in patients suffering from epiphora as it may not cause significant overall change in health status or disability. They developed the Lac-Q questionnaire, which includes questions for eye-specific symptoms as well as overall social impact and demonstrated its validity and reliability in assessing patient-reported outcomes following DCR. Similar results were found by Ali et al. ${ }^{19}$

In our study, in addition to DCR outcomes, we aimed to assess and compare other common interventions for epiphora, including lid-tightening procedures and punctoplasty, and investigate their effect on healthrelated QoL. Therefore, we devised a questionnaire that combined a subjective epiphora severity scale (Munk score), as well as the general QoL questionnaire (GBI) and the social impact questions from the validated Lac-Q questionnaire.

Our questionnaire completion rate of $75 \%$ was comparable to that reported by Smith et al. ${ }^{5}$ Overall, we found a significant improvement in post-interventional change in patients' reported severity of epiphora as well as QoL benefit in all four intervention groups. Pre-operative mean Munk score was very similar across all groups, ranging from 3.43 for lid tightening to 3.56 for DCR, and a significant reduction in Munk score postoperatively was noted in all intervention groups $(P<0.001)$. Similarly, the 95\% CI for the mean total GBI score did not span the 'no change' (or 'zero') point for any of the four groups, suggesting the positive patient benefit gained through these procedures was unlikely to be due to chance.

Our results show the greatest improvement in severity of tearing and perceived patient benefit in the DCR group, with mean post-op Munk score of 0.48 and total GBI of +42.67 . Unlike the other three groups where a few negative total GBI scores were recorded, all patients perceived some benefit post DCR as suggested by minimum total GBI score of +11.1 . Previous reports in the literature demonstrate similar findings, with DCR patients generally scoring higher on GBI compared to other oculoplastic interventions. Our GBI results post lidtightening surgery $(+19.65)$, punctoplasty $(+16.06)$, and the two interventions combined $(+26.53)$ are comparable to previously reported findings of $+21.36^{\text {(ref. } 15)}$ and $+24.89^{\text {(ref. 5) }}$ for ptosis, $+17.68^{\text {(ref. 5) }}$ for ectropion and $+25.25^{\text {(ref. 5) }}$ for entropion, and $+29.2^{\text {(ref. 16) }}$ post botulinum toxin for blepharospasm. Our DCR outcomes show 
a
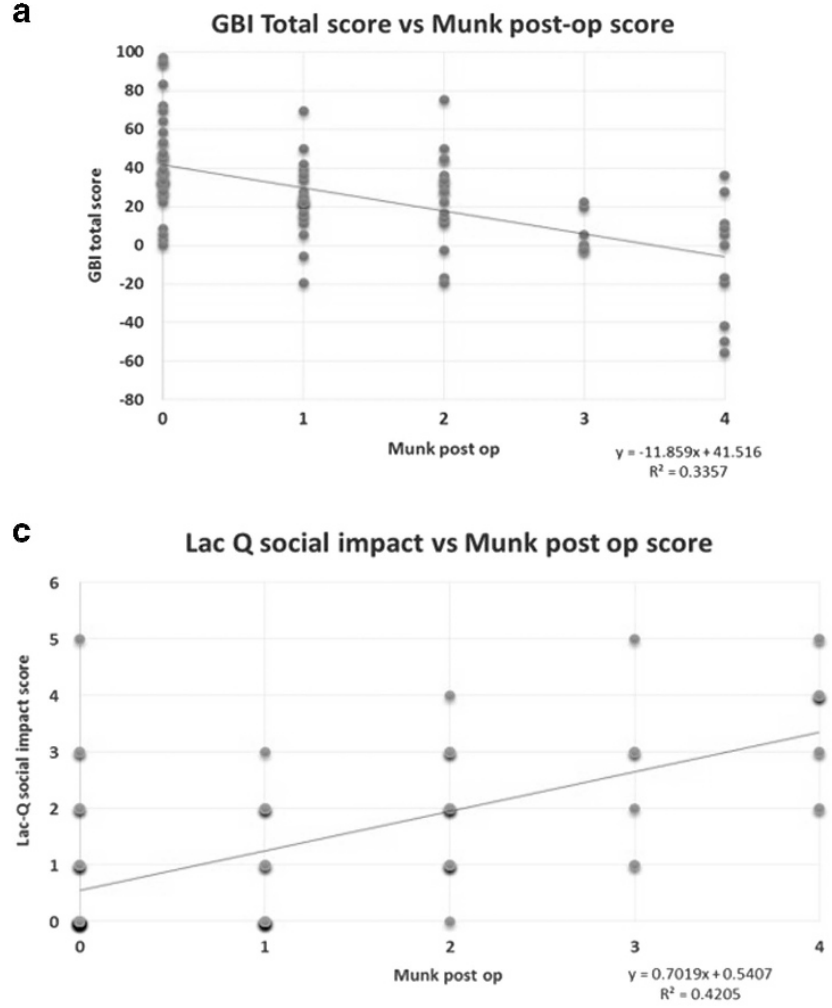

b

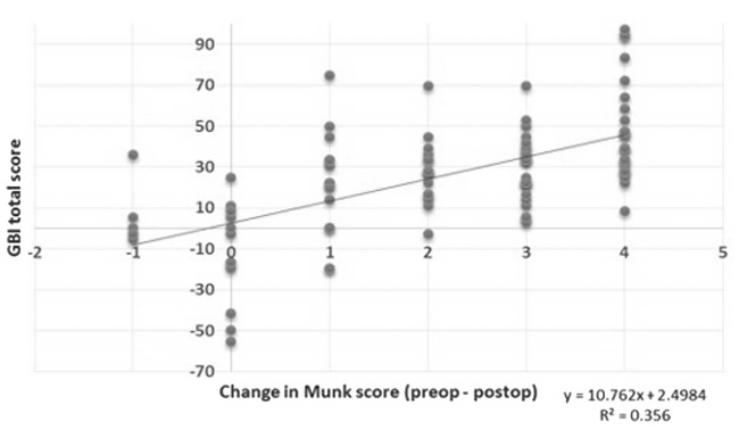

d

d Lac Q social impact score vs change in Munk score

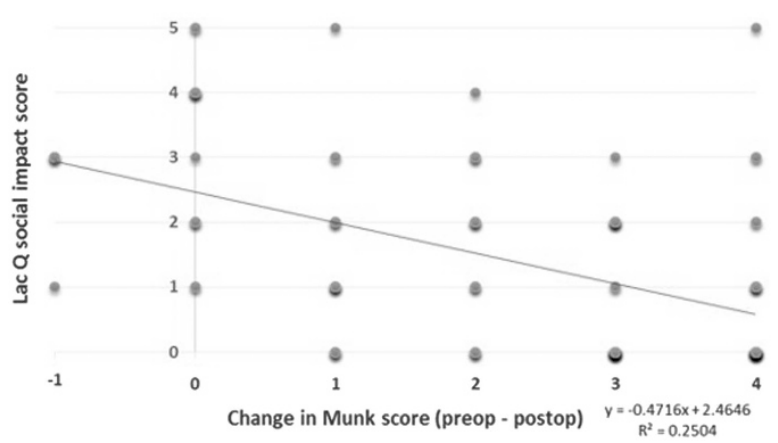

e

GBI total score vs Lac-Q post op

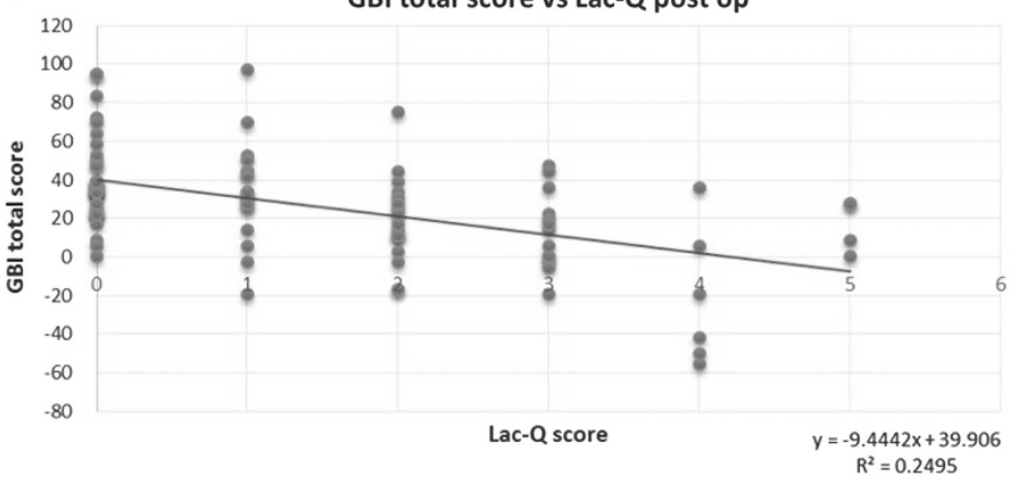

Figure 4 Linear regressions. (a) GBI total score vs Munk post-op score for all patients. (b) GBI total score vs change in Munk score (preop minus post-op score) for all patients. (c) Lac-Q 'social score' post op vs Munk post op score for all patients. (d) Lac-Q 'social score' post op vs change in Munk score for all patients. (e) GBI Total score vs Lac-Q post op score for all patients.

slightly higher levels of patient benefit compared to other published studies, which range from $+16.01^{\text {(ref. } 8)}$ to $+32.25^{\text {(ref. 5) }}$ post external and $+15.04^{\text {(ref. } 4)}$ to +37 at 2 months increasing to +52 at 6 months post-endonasal DCR. ${ }^{10}$

The Lac-Q questionnaire, validated for DCR patients by Mistry et al, ${ }^{11}$ has only been utilised in one published study by Ali et al ${ }^{19}$ so far. They reported improvement in endoscopic DCR patients' Lac-Q social impact score from 3.88 preoperatively to 0.5 at 4 weeks and 0.3 at 16 weeks post surgery, which is slightly lower than our finding of 1.05 post DCR.
The majority of previously published studies investigating patient benefit post lacrimal surgery are retrospective, ${ }^{1,4-6}$ the prospective design of our study allowed for assessment of the patient's severity of epiphora (Munk score) shortly prior to surgical intervention, as well as postoperatively at the time of completing the GBI and Lac-Q 'social impact' questionnaire. We found statistically significant correlation between the patients' subjective improvement in tearing and both the GBI and Lac-Q 'social score.'

There are many causes of epiphora, including obstruction (at the level of punctum, canaliculus, lacrimal 
sac or nasolacrimal duct), eyelid malposition or laxity, lacrimal pump failure, and reflex tearing. Sibley et al ${ }^{20}$ have reported multi-factorial aetiology of epiphora in $28.7 \%$ of patients presenting to a specialist ophthalmic service with tearing. Identifying the precise cause for epiphora is sometimes difficult, and in some cases can only be confirmed in a step-wise approach to eliminate each factor. In patients with multi-factorial epiphora presenting to our service, we choose to perform what could be considered the 'simpler' procedure such as punctoplasty or lid tightening first before undergoing more complex surgery like DCR, especially in the more elderly and frail patients. We only included patients undergoing their first surgical intervention for epiphora in this study, and it is likely that some of those who underwent lid tightening or punctoplasty may also have an element of nasolacrimal duct obstruction, requiring further surgical intervention.

Our punctoplasty group had the lowest mean GBI score but the greatest standard deviation and, as seen in Figure 2, there was a small proportion of punctoplasty patients with particularly negative scores causing positive skew. Post-operative clinic visit assessment for these two patients, who scored -55.56 and -41.67 , revealed significant additional causes for watering with ocular surface disease and previously undetectable nasolacrimal duct obstruction, respectively. Both had patent puncti following punctoplasty surgery but required further treatment, including DCR surgery in the latter case.

Although we found clear correlations between levels of watering and the questionnaire responses, we also noted some potential limitations. For example, patients who reported no watering postoperatively (Munk score $=0$ ) had a range of total GBI scores from 0 to +97.22 as shown in Figure 4a. Similarly, patients who reported no change in severity of tearing postoperatively (change in Munk score $=0$ ) had scores ranging from -55.56 to +25 (Figure $4 \mathrm{~b}$ ). In some cases of severe epiphora, the Munk scale may be unhelpful. For example, a patient whose frequency of wiping improved from over 50 times a day preoperatively to 12 times a day postoperatively reported significant benefit from surgery but had a Munk score of 4 both pre- and post surgery.

Similarly, a potential weakness of the GBI questionnaire is that it is non-specific, and a negative score may not indicate an adverse outcome from surgery but rather be due to general poor health or other illness. In addition, inconsistencies in GBI scores have been noted by Smirnov et al, ${ }^{10}$ who found misunderstanding of GBI questions by some patients, and the questionnaire was difficult to fill out for a few elderly patients without further guidance. They suggested the use of simplified, more lacrimal symptom-specific, questionnaires such as the NLDO-SS, which has been validated post DCR.
However, there is no specific lacrimal PROM questionnaire available that has been validated in nonDCR surgical interventions for epiphora. On the basis of our results, a combination of Munk score and Lac-Q social impact score both pre- and postoperatively could act as a simple and effective tool for assessing patient benefit following surgical interventions for epiphora; however, further work is needed to create a validated PROM questionnaire.

\section{Conclusions}

Reliable PROM tools are needed to confirm improvement in symptoms and patients' general well-being after lacrimal surgery as a successful anatomical surgical outcome does not necessarily confer an improvement in QoL or patient satisfaction. We have demonstrated significant patient-reported benefit and improvement in symptoms following the commonly performed oculoplastic interventions for epiphora, of DCR, lid tightening, and punctoplasty. Further work is required to design and validate a PROM questionnaire for surgical interventions for epiphora other than DCR.

\section{Summary}

What was known before

- The Glasgow benefit inventory questionnaire has been validated for assessment of patient-reported benefit following several oculoplastic procedures and a number of studies have demonstrated patient benefit and improvement in QoL following DCR surgery.

What this study adds

- We have demonstrated significant patient-reported QoL improvements in patients undergoing DCR, lid tightening, and punctoplasty surgery for epiphora.

- Patient-reported outcome measure questionnaires are a useful tool to demonstrate, and compare patient benefit and effectiveness of oculoplastic interventions.

\section{Conflict of interest}

The authors declare no conflict of interest.

\section{References}

1 Bakri SJ, Carney AS, Robinson K, Jones NS, Downes RN. Quality of life outcomes following dacryocystorhinostomy: external and endonasal laser techniques compared. Orbit 1999; 18(2): 83-88.

2 Spielmann PM, Hathorn I, Ahsan F, Cain AJ, White PS. The impact of endonasal dacryocystorhinostomy (DCR), on patient health status as assessed by the Glasgow benefit inventory. Rhinology 2009; 47(1): 48-50. 
3 Yeniad B, Uludag G, Kozer-Bilgin L. Assessment of patient satisfaction following external versus transcanalicular dacryocystorhinostomy with a diode laser and evaluation if change in quality of life after simultaneous bilateral surgery in patients with bilateral nasolacrimal duct obstruction. Curr Eye Res 2012; 37(4): 286-292.

4 Jutley G, Karim R, Joharatnam N, Latif S, Lynch T, Olver JM. Patient satisfaction following endoscopic endonasal dacryocystorhinostomy: a quality of life study. Eye 2013; 27(9): 1084-1089.

5 Smith HB, Jyothi SB, Mahroo OA, Shams PN, Sira M, Dey S et al. Patient-reported benefit from oculoplastic surgery. Eye 2012; 26(11): 1418-1423.

6 Feretis M, Newton JR, Ram B, Green F. Comparison of external and endonasal dacryocystorhinostomy. J Laryngol Otol 2009; 123(3): 315-319.

7 Ho A, Sachidananda R, Carrie S, Neoh C. Quality of life assessment after non-laser endonasal dacryocystorhinostomy. Clin Otolaryngol 2006; 31(5): 399-403.

8 Hii BW, McNab AA, Friebel JD. A comparison of external and endonasal dacryocystorhinostomy in regard to patient satisfaction and cost. Orbit 2012; 31(2): 67-76.

9 Ramey NA, Butt Z, Burkat CN, Ross JG, Lucarelli MJ. Patient-reported outcomes: comprehensive analysis for the oculofacial clinician. Ophthal Plast Reconstr Surg 2014; 30(4): 279-289.

10 Smirnov G, Tuomilehto H, Kokki H, Kemppainen T, Kiviniemi V, Nuutinen J et al. Symptom score questionnaire for nasolacrimal duct obstruction in adults-a novel tool to assess the outcome after endoscopic dacryocystorhinostomy. Rhinology 2010; 48: 446-451.

11 Mistry N, Rockley TJ, Reynolds T, Hopkins T. Development and validation of a symptom questionnaire for recording outcomes in adult lacrimal surgery. Rhinology 2011; 49: 538-545.

12 Munk PL, Lin DT, Morris DC. Epiphora: treatment by means of dacryocystoplasty with balloon dilatation of the nasolacrimal drainage apparatus. Radiology 1990; 177: 687-690.

13 Robinson K, Gatehouse S, Browning GG. Measuring patient benefit from otorhinolaryngological surgery and therapy. Ann Otol Rhinol Laryngol 1996; 105(6): 415-422.

14 Mahroo OA, Hysi PG, Dey S, Gavin EA, Hammond CJ, Jones CA. Outcomes of ptosis surgery assessed using a patient-reported outcome measure: an exploration of time effects. Br J Ophthalmol 2014; 98(3): 387-390.

15 Maycock N, MacGregor C, Saunders DA, Parkin B. Long term patient-reported benefit from ptosis surgery. Eye 2015; 29: $872-874$.

16 MacAndie K, Kemp E. Impact on quality of life of botulinum toxin treatments for essential blepharospasm. Orbit 2004; 23: 207-210.

17 Medical Research Council UK Institute of Hearing. Glasgow Health Status Questionnaires (GBI \& GHSI). Available at: <https://www.ihr.mrc.ac.uk/projects/gbi> (accessed 27 July 2016).

18 Kafil-Hussain N, Khooshabeh R. Clinical research, comparison of subjective visual function in patients with epiphora and patients with second-eye cataract. Orbit 2005; 24: 33-38.

19 Ali MJ, Iram S, Ali MH, Naik MN. Assessing the outcomes of powered endoscopic dacryocystorhinostomy in adults using the lacrimal symptom (Lac-Q) questionnaire. Ophthal Plast Reconstr Surg 2016; 33(1): 65-68.

20 Sibley D, Norris JH, Malhotra R. Management and outcomes of patients with epiphora referred to a specialist ophthalmic plastic unit. Clin Exp Ophthalmol 2013; 41(3): 231-238.

Supplementary Information accompanies this paper on Eye website (http://www.nature.com/eye) 